\title{
Towards a Reconceptualization of the Temporoparietal Junction in Stimulus-Driven Attention
}

\author{
Charles J. Lynch \\ Department of Psychology, Georgetown University, Washington, DC 20057 \\ Review of Han and Marois
}

Adaptive behavior requires orienting attention to unexpected but potentially important stimuli in our environment. Consider, for example, a man driving a car when a light appears on the dashboard. This event is unexpected, leading the man to detect and orient to the warning light. The man then determines that the light indicates fuel is low and chooses an appropriate course of action, for example, immediately searching for a service station. This simple illustration highlights that the process of responding to stimuli outside focused attention, termed stimulus-driven attention, involves behaviorally dissociable subprocesses: stimulus detection/orienting and stimulus evaluation.

A recent study by Han and Marois (2014), published in The Journal of Neuroscience, argues that two dominant neurocognitive models of stimulus-driven attention highlight detecting and orienting to unexpected stimuli without adequately addressing how evaluative processes are implemented and by which brain regions. One model, based on findings of taskevoked functional magnetic resonance (fMRI) activation studies, proposes that a right lateralized ventral attention network, consisting of the temporoparietal junction (TPJ) and inferior frontal junc-

Received July 17, 2014; revised Aug. 18, 2014; accepted Aug. 20, 2014. I thank Dr. Chandan Vaidya for her excellent feedback on this Journal Club. Correspondence should be addressed to Charles J. Lynch, 403B WhiteGravenor, Department of Psychology, Georgetown University, Washington, DC 20057. E-mail: c1968@georgetown.edu.

DOI:10.1523/JNEUROSCI.2996-14.2014

Copyright $\odot 2014$ the authors $\quad 0270-6474 / 14 / 3413277-02 \$ 15.00 / 0$ tion (IFJ) (Corbetta et al., 2008), is sensitive to behaviorally relevant stimuli that violate expectations. The second model, based on resting-state fMRI connectivity work, describes a salience network consisting of the anterior insular (AI) and dorsal anterior cingulate cortex (dACC) (Seeley et al., 2007) that responds to behaviorally important stimuli and initiates appropriate cognitive control. These two systems are often considered independently of each other, despite apparent overlap in their putative functions, exacerbating uncertainty regarding the role of individual regions. In particular, the role of the TPJ has been debated (Geng and Vossel, 2013).

The TPJ was originally posited as a "circuit breaker" that reorients focused attention, facilitated by a dorsal attention network (DAN), when unexpected stimuli (i.e., oddballs) are detected (Corbetta et al., 2008). However, evidence supporting this stimulus detection/reorienting role of the TPJ has been mixed (Diquattro et al., 2013). A recently proposed alternative is that the TPJ serves a more domaingeneral role of updating task representations (Geng and Vossel, 2013). This view fits well the observation that the taskrelevance of unexpected stimuli, regardless of sensory salience, drives TPJ activation (Corbetta et al., 2008). The data presented by Han and Marois (2014) can further theoretical frameworks of the TPJ.

Traditional fMRI oddball paradigms, which require responding to a rare target amid more familiar stimuli, present stimuli too briefly $(<1 \mathrm{~s})$ to distinguish neural responses associated with stimulus detection/orienting from those involved in stimulus evaluation. To overcome this obstacle, a significant innovation by Han and Marois (2014) was the implementation of a temporally extended oddball task. Subjects were instructed to look for a target image among a stream of distractors. Unbeknown to subjects, $10 \mathrm{~s}$ movies (i.e., the oddball) appeared unexpectedly during the task. Blood-oxygen level-dependent (BOLD) responses to oddball trials were measured within core nodes of the ventral attention network (TPJ and IFJ) and the salience network (AI and dACC).

The authors hypothesized that nodes involved in stimulus detection/orienting would demonstrate transient responses at oddball onset/offset (e.g., a dual peak response), whereas nodes contributing to stimulus evaluation would demonstrate sustained activation (e.g., a single peak response). In addition, the authors predicted that by increasing oddball familiarity in a second run, stimulus-evaluation regions would have a diminished BOLD response, whereas the response of regions involved in stimulus detection/orienting would remain unchanged. The rationale was that while a familiar oddball requires less evaluation, the onset/offset of the oddball remains unexpected. Using this simple approach, the authors examined BOLD response profiles of individual network nodes, including the TPJ, to infer roles in either stimulus detection/orienting or stimulus evaluation.

Remarkably, only the TPJ demonstrated a sustained single-peak BOLD re- 
sponse that was attenuated in the second run, indicative of a unique evaluative role. In contrast, the AI and AACC were sensitive to the onset/offset of oddball trials, but not oddball content. As the authors note, this is consistent with previous accounts that the AI and ACC form a salience network (Seeley et al., 2007). The BOLD response profile of the IFJ changed between runs, from a single-peak to a dual-peak response. The authors argued that this suggests a dual role for the IFJ, supporting both orienting and evaluative processes.

A striking finding by Han and Marois (2014) was that the primary role of the TPJ is distinct from stimulus detection/ orienting, which was more closely associated with the salience network and IFJ. Instead, the authors propose that the TPJ evaluates unexpected stimuli, presumably to determine factors such as task relevance (Corbetta et al., 2008). It is notable that this proposal does not preclude a role of the TPJ in stimulus-driven attention. It does, however, reconcile why the TPJ is recruited during cognitive tasks unrelated to attentional orienting that involve some form of stimulus evaluation (e.g., inferring the mental states of others and understanding biological motion). This domain-general account must be weighed alongside findings of functional heterogeneity within the TPJ, which raises the alternative possibility that distinct subregions contribute to different domains of cognition (Mars et al., 2012).

A critical issue for future research is elucidating mechanisms underlying TPJmediated stimulus evaluation. Two questions may be informative in exploring this issue. First, how are patterns of TPJ connectivity modulated during periods of stimulus evaluation? Work examining functional connectivity by measuring synchronization in fMRI signals between brain regions during specific task conditions can help address this question. One theory is that the TPJ receives signals from DAN nodes to facilitate appropriate attentional control (Corbetta et al., 2008). Two recent studies found that signals from the DAN to the TPJ are indeed modulated when evaluating visual cues (Diquattro et al., 2013) and that this may facilitate task performance (Wen et al., 2012). However, these studies used region-of-interest-based approaches, constraining analyses to the TPJ and a priori defined nodes of the DAN. This prevented testing whether additional regions interact with the TPJ as well.

As Han and Marois (2014) point out, stimulus evaluation may extend beyond purely attentional processes. It is plausible then to also consider interactions between the TPJ and brain regions responsible for object identification/discrimination (e.g., occipitotemporal cortex and perirhinal cortex; Murray and Richmond, 2001) and those maintaining representations relevant for the task at hand (e.g., medial temporal lobe; Cabeza et al., 2011). There is some evidence that structural connections may exist between the TPJ and these regions of the brain. For example, axonal tracing in nonhuman primates has identified connections between ventral parietal cortex (a possible homolog of human TPJ) and ventral occipitotemporal, medial temporal, and prefrontal cortex (Petras, 1971). Although this hypothesis is speculative, future work examining whole-brain patterns of TPJ functional connectivity during periods of stimulus evaluation can test it.

Second, what are the neurochemical contributions to TPJ-mediated stimulus evaluation? Although several neuromodulators, including norepinephrine (Dayan and $\mathrm{Yu}, 2006$ ), have been broadly linked with stimulus-driven attention, a specific role in TPJ function is unclear. Nonhuman primate work has found that efferent projections from the locus ceruleus, a primary site of norepinephrine synthesis, terminate in inferior parietal and posterior superior temporal cortex (Nieuwenhuis et al., 2005). This highlights human temporoparietal cortex as a potential target of norepinephrine innervation. Consist with this notion, administrating $\beta$-adrenergic blocking drugs in humans reduces the TPJ BOLD response to oddball stimuli (Strange and Dolan, 2007).

Interestingly, a recent fMRI study raises the possibility that noradrenergic projections contribute to TPJ function when making decisions dependent on stimulus evaluation (Kahnt and Tobler, 2013). In this study, subjects learned associations between visual cues and possible outcomes, both positive and negative. In a subsequent fMRI task, subjects either accepted or rejected an "offer" based on the outcome associated with a cue. Critically, not only was the TPJ engaged in this task, but also functional connectivity between the TPJ and locus ceruleus was modulated by the salience (i.e., task relevance in terms of the expected outcome) of the cue (Kahnt and Tobler, 2013). This last point builds on the model proposed by Han and Marois (2014) by providing a potential neurochemical mechanism for the TPJ in a nonattention task context that still requires stimulus evaluation.
In summary, Han and Marois (2014) provide preliminary evidence for a stimulus evaluation hypothesis of the TPJ, consistent with other recent lines of evidence challenging traditional accounts of attentional reorienting in favor of a more domain-general function (Geng and Vossel, 2013). Although the precise brain mechanisms underlying this role of the TPJ remain unclear, they will become clearer as this alternative model of the TPJ is refined.

\section{References}

Cabeza R, Mazuz YS, Stokes J, Kragel JE, Woldorff MG, Ciaramelli E, Olson IR, Moscovitch M (2011) Overlapping parietal activity in memory and perception: evidence for the attention to memory model. J Cogn Neurosci 23:32093217. CrossRef Medline

Corbetta M, Patel G, Shulman GL (2008) The reorienting system of the human brain: from environment to theory of mind. Neuron 58: 306-324. CrossRef Medline

Dayan P, Yu AJ (2006) Phasic norepinephrine: a neural interrupt signal for unexpected events. Network 17:335-350. CrossRef Medline

Diquattro NE, Sawaki R, Geng JJ (2013) Effective connectivity during feature-based attentional capture: evidence against the attentional reorienting hypothesis of TPJ. Cereb Cortex 31: 18026-18035. CrossRef Medline

Geng JJ, Vossel S (2013) Re-evaluating the role of TPJ in attentional control: contextual updating? Neurosci Biobehav Rev 37:26082620. CrossRef Medline

Han SW, Marois R (2014) Functional fractionation of the stimulus-driven attention network. J Neurosci 34:6958-6969. CrossRef Medline

Kahnt T, Tobler PN (2013) Salience signals in the right temporoparietal junction facilitate value-based decisions. J Neurosci 33:863869. CrossRef Medline

Mars RB, Sallet J, Schüffelgen U, Jbabdi S, Toni I, Rushworth MF (2012) Connectivity-based subdivisions of the human right temporoparietal junction area: evidence for different areas participating in different cortical networks. Cereb Cortex 22:18941903. CrossRef Medline

Murray EA, Richmond BJ (2001) Role of perirhinal cortex in object perception, memory, and associations. Curr Opin Neurobiol 11:188-193. CrossRef Medline

Nieuwenhuis S, Aston-Jones G, Cohen JD (2005) Decision making, the P3, and the locus coeruleus-norepinephrine system. Psychol Bull 131:510-532. CrossRef Medline

Petras JM (1971) Connections of the parietal lobe. J Psychiatr Res 8:189-201. CrossRef Medline

Seeley WW, Menon V, Schatzberg AF, Keller J, Glover GH, Kenna H, Reiss AL, Greicius MD (2007) Dissociable intrinsic connectivity networks for salience processing and executive control. J Neurosci 27:2349-2356. CrossRef Medline

Strange BA, Dolan RJ (2007) Beta-adrenergic modulation of oddball responses in humans. Behav Brain Funct 3:29. CrossRef Medline

Wen X, Yao L, Liu Y, Ding M (2012) Causal interactions in attention networks predict behavioral performance. J Neurosci 32:1284-1292. CrossRef Medline 